\title{
Blood Loss through AV Fistula: A Case Report and Literature Review
}

\author{
Fahad Saeed, Nadia Kousar, Ramapriya Sinnakirouchenan, Vijaya S. Ramalingam, \\ Philip B. Johnson, and Jean L. Holley
}

College of Medicine at Urbana-Champaign, University of Illinois, 611 W Park Street, Urbana, IL 61801, USA

Correspondence should be addressed to Fahad Saeed, fahadsaeed20@gmail.com

Received 3 January 2011; Revised 10 March 2011; Accepted 24 March 2011

Academic Editor: Greg Tesch

Copyright (C) 2011 Fahad Saeed et al. This is an open access article distributed under the Creative Commons Attribution License, which permits unrestricted use, distribution, and reproduction in any medium, provided the original work is properly cited.

\begin{abstract}
Little has been written about acute blood loss from hemodialysis vascular access. We describe a 57 -year-old Caucasian male with an approximately $7 \mathrm{gm} / \mathrm{dL}$ drop in hemoglobin due to bleeding from a ruptured aneurysm in his right brachiocephalic arteriovenous fistula (AVF). There was no evidence of fistula infection. The patient was successfully managed by blood transfusions and insertion of a tunneled dialysis catheter for dialysis access. Later, the fistula was ligated and a new fistula was constructed in the opposite arm. Aneurysm should be considered in cases of acute vascular access bleeding in chronic dialysis patients.
\end{abstract}

\section{Introduction}

Acute blood loss through vascular access can be a life threatening problem. The case under discussion highlights the importance of vascular access monitoring and surveillance to prevent failure. Blood loss through AVF can be from aneurysms, stenosis and subsequent rupture, infection, trauma, suicide and, use of anticoagulants and antiplatelet drugs. Common causes of blood loss through the AV fistula in ESRD patients are also reviewed in the discussion part.

\section{Case}

A 57-year-old Caucasian man with a past medical history of benign essential hypertension, dyslipidemia, coronary artery disease status post 1 coronary artery bypass grafting, systolic congestive heart failure secondary to ischemic cardiomyopathy, type 1 diabetes mellitus with associated complications of diabetic retinopathy, neuropathy and nephropathy, h/o ischemic stroke, Human Immunodeficiency Virus (HIV) positivity since 1992, and end-stage renal disease on hemodialysis through a right arm brachiocephalic AV fistula (AVF) created approximately five years ago presented to the emergency room (ER) with bleeding from his AVF. He started having problems with this AV fistula almost a year previously when he underwent an AV fistulogram due to decreased dialysis clearance. The fistulogram identified two areas of venous stenosis and an aneurysmal dilatation in the proximal portion of the outflow vein. No central vein stenosis was seen. Successful angioplasty of the two sequential stenoses was performed with establishment of good fistula flow. Five months after this procedure, prolonged fistula bleeding after a routine dialysis led to another AV fistulogram which demonstrated a recurrent stenosis of the outflow tract in the cephalic vein. Angioplasty was again successfully performed with no visible residual stenosis on postoperative fistulogram. No routine access flow monitoring was performed in the dialysis unit. No significant dialysis access issues were observed until two weeks before the current presentation when dialysis nurses observed aneurysmal dilatation of his AV fistula and recurrent prolonged bleeding from the cannulation site. Vascular surgery appointment was scheduled and pending. His home medications included aspirin $81 \mathrm{mg}$, clopidogrel $75 \mathrm{mg}$, lamivudine, atazanavir, lanthanum carbonate, carvedilol, amlodipine, valsartan, levothyroxine, insulin aspart, and lantus. Of note, he was on clopidogrel because of ischemic stroke while being on aspirin. He was on a combination of both antiplatelets for the last 4 years. On the day of presentation, an uncomplicated hemodialysis treatment concluded at $11 \mathrm{AM}$ and the patient 


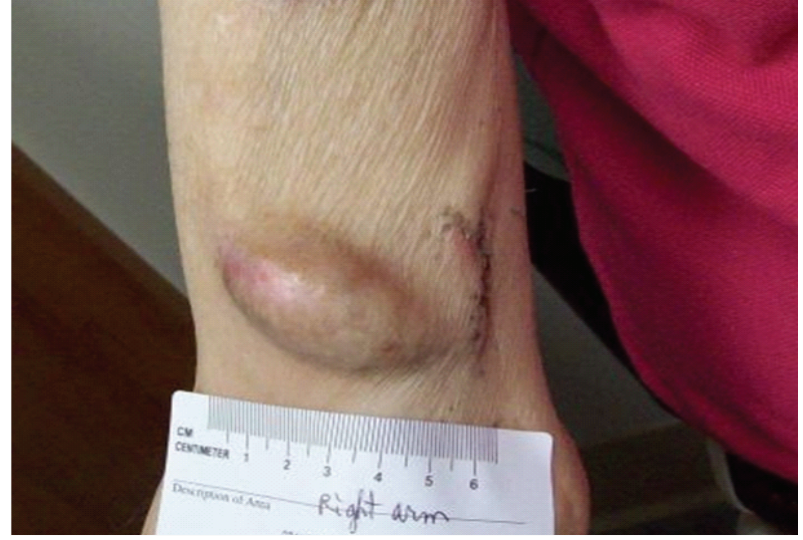

FIGURE 1: Right upper arm AV fistula aneurysm of the patient. Size is approximately $6 \times 3 \mathrm{~cm}$.

drove himself home. About 30 minutes after his dialysis treatment ended, he called a friend and complained of bleeding from his AVF. The friend arrived half an hour later and called 911. The patient was coherent and conscious but complaining of generalized weakness, fatigue, and shortness of breath. Emergency medical services responded and applied a pressure dressing to his AVF. Upon arrival in the ER, his blood pressure was $117 / 98 \mathrm{mmHg}$, pulse 79, and regular, respiratory rate 18, temperature $98.6 \mathrm{~F}, \mathrm{SpO} 292 \%$ on room air. He was pale and there was an area of aneurysmal dilatation of the right AVF with a pinpoint area of bleeding which had largely stopped with pressure. Patient's AVF is shown in Figure 1. A short arm cast was on the right wrist (wrist fracture had occurred three weeks earlier). There were no obvious signs and symptoms of infection of the AVF and no recent antibiotic use. Initial labs were remarkable for $\mathrm{Hgb}$ $6.7 \mathrm{gm} / \mathrm{dL}$ and Hct of $21.8 \%$. Of note, his hemoglobin the prior month was $13.2 \mathrm{gm} / \mathrm{dL}$. His PTT, INR, and platelets were normal. The patient was transfused 5 units of packed red blood cells with appropriate response in hemoglobin. Blood cultures remained negative. A tunneled catheter was placed for hemodialysis access. A new left arm basilic vein transposition AVF was created along with ligation of the right brachiocephalic AVF. Three months later, the right brachiocephalic fistula was removed by vascular surgery; intraoperatively a pseudoaneurysm and thrombosis of the AV fisula were observed. His hemoglobin six months after discharge is stable at $12.5 \mathrm{gm} / \mathrm{dL}$.

\section{Discussion}

The Centers for Medicare and Medicaid Services Interpretive Guidance Update requires every dialysis facility to have an ongoing program for vascular access monitoring and surveillance for early detection of failure and to allow timely referral of patients for intervention. Physical examination can be used as a monitoring tool to exclude low flows associated with impending vascular access failures. There are 3 components to the access examination: inspection (look),
TABLE 1: Causes of blood loss through AV fistula.

Causes of blood loss through AV fistula

(1) Aneurysm formation

(2) Stenosis and subsequent rupture

(3) Infection

(4) Trauma

(5) Use of anticoagulants and antiplatelets

(6) Suicide

palpation (touch), and auscultation (listen). Simple inspection can reveal the presence of aneurysms. A fistula that does not at least partially collapse with arm elevation is likely to have an outflow stenosis. Strictures can be palpated and the intensity and character of the bruits can suggest the location of stenosis. Downstream stenosis also produces an overall dilation of the vein, giving it "aneurysmal" proportions. Observance of changes in adequacy or in pressures measured during dialysis, difficulties in cannulation, or in achieving hemostasis may also be used as monitoring tools [1-4].

Surveillance strategies include device-based methods such as access flow measurements, direct or derived static venous pressure ratios, and duplex ultrasound, and so forth. However, the proper role of surveillance awaits the results of further research. The best available evidence indicates that fistula surveillance does not prolong fistula life and there is limited evidence that surveillance may reduce fistula thrombosis [4]. Serial monitoring of aneurysms may give the opportunity to potentially prevent rupture by timely intervention.

Evidence of periodic monitoring and surveillance of the vascular access should be documented on the dialysis treatment record, progress notes, or on a separate log. A member of the facility staff must review the vascular access monitoring/surveillance documentation to identify adverse trends and take action if indicated.

Our patient probably would have benefited from early vascular surgical intervention before an episode of life threatening hemorrhage. He was on both aspirin and clopidogrel although the current evidence suggests that the combined use of aspirin and clopidogrel does not offer greater benefit for stroke prevention than either agent alone but does substantially increase the risk of bleeding complications [5]. Thus, it is likely that the antiplatelet agents contributed to his bleeding and stopping the [6] dual antiplatelet therapy should have been considered when aneurysmal dilation of the fistula was initially noticed.

The most common sources of acute access blood loss in hemodialysis patients are shown in Table 1 . These will be briefly reviewed.

3.1. Aneurysms. Aneurysms usually occur in long-lasting arteriovenous fistulas and form in areas of repeated needle puncture sites [7]. Repeated needle punctures of the arterialized veins over time result in destruction of the venous wall and replacement by scar tissue causing loss of elasticity. Subsequently, thinning of the vessel wall increases the risk 
for rupture and bleeding [8]. There is also an increased risk of bleeding each time after insertion and removal of dialysis needles [9]. This is because the vessel wall is thinned in an aneurysm and is easily injured and prone to bleeding and rupture [10].

Cannulation should not be continued along any type of aneurysm, particularly in patients for whom the skin layer within the aneurysm is thin and prone to infection, a sign of impending perforation [11].

Long-standing high blood flow rates through an $\mathrm{AV}$ fistula results in shear forces that cause damage to the elastic fibers of the internal elastic lamina. The high flow rates and the resulting shear forces from repeated dialysis treatments limit time for healing, thereby causing destruction of the venous wall and aneurysm formation [12].

Arteriovenous fistula aneurysms may be false aneurysms or venous aneurysms [13]. False aneurysms are hematomas located outside the vessel wall, formed due to a leaking hole in the artery and are most often due to iatrogenic trauma, primarily repeated needle punctures. Infection of a false aneurysm increases the risk of rupture and bleeding as the vessel wall is already thin.

Risk for bleeding and hematoma formation is greatest in the early stages of use of a fistula and greater in brachiobasilic fistulae than fistulae at the wrist or elbow [11]. Venous aneurysms occur when repeated trauma to the venous wall is coupled with complete stenosis. The increased venous resistance leads to progressive enlargement of the venous aneurysm, thus increasing the risk of rupture [13]. True aneurysms are fusiform, develop slowly over several years, and lie beneath intact, nonulcerated skin making them less prone to infection and rupture. On the contrary, pseudoaneurysms are usually saccular with thinning of overlying skin and develop over a short period of time, making them more prone to rupture and infection [14]. Aneurysms are at risk for rupture if they are large and rapidly expanding, if there is thinning of overlying skin exposing the underlying fistula and if there are signs of local infection $[15,16]$.

The occurrence of large aneurysms, in addition to threatening the function of the AVF, may ultimately rupture with catastrophic sequelae. However surgical options to repair such large aneurysms are limited and often involve loss of the AVF. Endovascular procedures such as angioplasty and stenting have been increasingly used to treat vascular access dysfunction and to prolong the life of an AVF [17].

3.2. Stenosis. Venous stenosis within the perianastomotic region is the leading cause of vascular access dysfunction [18]. The basic underlying mechanism of venous stenosis is neointimal hyperplasia due to cytokine production, cellular proliferation, and microvessel formation [19]. This is triggered by high intraluminal pressure, turbulent blood flow, vascular calcification, endothelial injury, and increased levels of fibronectin [20, 21]. Development of stenosis of AV fistula is also influenced by venous capacitance, surgical technique, and the site of the AV fistula.

In hemodialysis patients, a native $\mathrm{AV}$ fistula is the vascular access of choice owing to its superior patency and low complication rates. Venous stenosis per se does not lead to blood loss but can be complicated by aneurysm formation with subsequent rupture and blood loss.

Aneurysms are observed within the first postanastomotic venous segment in the presence of a hemodynamically relevant stenosis in the juxta-anastomotic position The therapy of choice is a new AV anastomosis using a healthy venous segment located a few centimeters more proximally, but as close to the former anastomosis as possible, to preserve the maximum area for cannulation.

3.3. Infections. Vascular access site infection is considered to be the most challenging complication and the major cause of morbidity and mortality among chronic hemodialysis patients [22]. It is also an important cause for AV fistula failure. The source of sepsis in the dialysis population is largely related to vascular access. Infection rate is highest with temporary central vein catheter followed by tunneled central vein catheter and prosthetic AV grafts and is least with primary AV fistulas [23-27]. Common factors associated with increased AVF and AV graft infection include prior episode of bacteremia, immunosuppression, poor personal hygiene, poor aseptic techniques during cannulation, repeated cannulations, defects in neointimal lining, nasal staphylococcus aureus carriage, diabetes mellitus, uremia, and age of fistula $[22,28]$. Blood loss due to infections of AV fistulas results from vessel wall weakening leading to aneurysm formation and rupture. Preexisting aneurysms can rupture secondary to superimposed infections. Surgical debridement of localized infections can also result in significant blood loss. Utilization of antibacterial soap followed by either $70 \%$ alcohol or $10 \%$ povidone iodine has been shown to reduce the risk of infections from needle cannulations [9]. A study showed a slightly greater incidence of AV fistula infections with the button hole technique for cannulation when compared with the rope ladder technique [29]. Recognizing and correcting the modifiable risk factors for infection will help minimize the incidence of AV fistula infection and thereby decrease the risk of access blood loss due to aneurysm rupture.

3.4. Trauma. The most common trauma to AV fistulas is repeated needle cannulations during dialysis. Dialysis technicians and patients are taught to rotate needle insertion sites during each dialysis. Proper cannulation is a vital and fundamental skill that every hemodialysis nurse should master. First cannulation should be carried out by nurses who have great expertise $[11,30]$.

To reduce the bleeding time after needle withdrawal, all superficial AV fistulas should be cannulated at an angle of 25 degrees [31]. There are 3 different cannulation techniques which include rope ladder, button hole, and area puncture.

In the rope ladder technique, puncture sites are spread along the length of the vascular access, starting distally, and proceeding proximally. This is the most popular method. The button hole technique consists of puncturing the same site, in the same direction and at the same angle and depth. The initial few punctures are made using a sharp needle 
and once a tract is formed, blunt needles are used. The area puncture technique involves needle insertion within a limited area of the fistula. This technique is associated with more complications such as aneurysm formation, thinning of the skin at puncture sites, increased risk of bleeding along the needles, and longer bleeding time after needle withdrawal [31].

A standard part of patient teaching should be on managing interdialytic bleeding that includes compression with a clean cloth and calling the dialysis facility for instructions. They should also be instructed to go to the Emergency Room or call 911 for a more severe, uncontrollable bleed. All patients should be taught to compress a bleeding access, wash skin over access with soap and water daily and before dialysis, and also recognize signs and symptoms of infection. All patients should know to avoid carrying heavy items draped over the access arm, avoid wearing occlusive clothing, wear protective clothing over the exposed fistula vein if working around machinery or sharp tools [11].

3.5. Use of Antiplatelets, Anticoagulants, and Bleeding Tendencies. Platelet biochemical abnormalities in ADP and serotonin compounds along with thromboxane A2 leading to defective platelet aggregation may contribute to increased bleeding tendencies in dialysis patients [32, 33]. Platelet numbers are usually not low enough to cause increased bleeding [34]. Factor 8 and Von Willebrand factor alteration may also play a crucial role as can anemia by leading to defective platelet aggregation as demonstrated by in vitro studies [35, 36]. Hemodialysis improves these hemostatic complications but use of heparin during dialysis may be a contributory factor to increased bleeding tendencies from dialysis access sites including petechial hemorrhages, blood blisters, ecchymosis, and hematoma. It is unusual for acute access blood loss to occur due to an underlying coagulopathy though theoretically it may prolong bleeding from any other reason.

Antiplatelet agents, such as dipyridamole and lowdose aspirin with or without sulfinpyrazone, aspirin plus clopidogrel, have been studied in an effort to decrease graft thrombosis but conflicting evidence exists in this regard and no real recommendations exist for the use of antiplatelet agents to prevent graft thrombosis $[37,38]$. In one clinical trial, role of aspirin plus clopidogrel versus double placebos was studied in preventing graft thrombosis [39], but the study was stopped because of a markedly increased risk of bleeding among those receiving active therapy (hazard ratio of 1.98, 95\% CI 1.19-3.28). At study end, dual antiplatelet therapy was not associated with a significant benefit in preventing thrombosis. Similarly, administration of warfarin does not increase graft survival and is associated with significant bleeding [40].

3.6. Suicide. The incidence and prevalence of depression among dialysis patients is unknown but estimates vary from 20 to 50 percent [41]. Approximately one-fourth of dialysis patients are depressed at some point in time. Furthermore, about 1 percent of the dialysis patients commit suicide, especially the elderly population [42]. Risk factors for suicide include male gender, a preexistent psychiatric diagnosis, and addiction to drugs or alcohol [43-45]. Bleeding from a vascular access is rarely a result of a suicide attempt.

\section{Conclusion}

In summary, patients with ESRD may lose blood from the AV fistula due to a variety of reasons. Aneurysms should be considered a risk factor for acute vascular access bleeding in chronic dialysis patients and should never be cannulated. If assessed to be at risk for rupture, a workup for associated access infection should be considered and a surgical consult for aneurysm repair initiated. Dual antiplatelet therapy should be avoided in patients with AV fistula aneurysms. Patient education is important to avoid complications of access bleeding; patients should be taught simple techniques like putting direct pressure to minimize bleeding while seeking acute medical care. Our patient should have applied direct pressure to his AV fistula and called for emergency services, thus potentially reducing the duration of his hospitalization.

\section{Conflict of Interests}

The authors declared that there is no conflict of interest.

\section{References}

[1] Centers for Medicare \& Medicaid Services (CMS) HHS, "Medicare and Medicaid programs; conditions for coverage for end-stage renal disease facilities. Final rule," Federal Register, vol. 73, pp. 20369-20484, 2008.

[2] Center for Medicaid and State Operations/Survey \& Certification Group: ESRD Interpretive Guidance Update, "Baltimore, MD: Department of Health Medicare and Medicaid Programs: Conditions for coverage for endstage renal disease facilities; final rule: rules and regulations, Part II," Tech. Rep. S\&C-0901, Department of Health and Human \& Human Services, Centers for Medicare \& Medicaid Services, 2008.

[3] W. D. Paulson and J. Work, "Controversial vascular access surveillance mandate," Seminars in Dialysis, vol. 23, no. 1, pp. 92-94, 2010.

[4] National Kidney Foundation, "KDOQI clinical practice guidelines for vascular access, update 2006," American Journal of Kidney Diseases, vol. 48, supplement 1, pp. S176-S247, 2006.

[5] H. C. Diener, J. Bogousslavsky, L. M. Brass et al., "Aspirin and clopidogrel compared with clopidogrel alone after recent ischaemic stroke or transient ischaemic attack in highrisk patients (MATCH): randomised, double-blind, placebocontrolled trial," Lancet, vol. 364, no. 9431, pp. 331-337, 2004.

[6] M. H. Usman, L. A. Notaro, R. Nagarakanti et al., "Combination antiplatelet therapy for secondary stroke prevention: enhanced efficacy or double trouble?" American Journal of Cardiology, vol. 103, no. 8, pp. 1107-1112, 2009.

[7] P. A. Mennes, L. A. Gilula, and C. B. Anderson, "Complications associated with arteriovenous fistulas in patients undergoing chronic hemodialysis," Archives of Internal Medicine, vol. 138, no. 7, pp. 1117-1121, 1978.

[8] "Hemodialysis vascular access and peritoneal dialysis access," Contributions to Nephrology, vol. 142, pp. 193-215, 2004. 
[9] "NKF-DOQI clinical practice guidelines for vascular access. National Kidney Foundation-Dialysis Outcomes Quality Initiative," The American Journal of Kidney Diseases, vol. 30, pp. S150-S191, 1997.

[10] N. S. Hakim, J. Romagnoli, J. C. Contis, J. Akoh, and V. E. Papalois, "Refashioning of an aneurysmatic arteriovenous fistula by using the multifire GIA 60 surgical stapler," International Surgery, vol. 82, no. 4, pp. 376-377, 1997.

[11] J. Gilmore, "KDOQI clinical practice guidelines and clinical practice recommendations-2006 updates," Nephrology Nursing Journal, vol. 33, no. 5, pp. 487-488, 2006.

[12] A. Sultana, F. Torella, R. McWilliams, and A. Bakran, "Axillary artery aneurysm following closure of haemodialysis fistula. A case report," Journal of Cardiovascular Surgery, vol. 48, no. 2, pp. 197-199, 2007.

[13] M. Haimov, A. Baez, M. Neff, and R. Slifkin, "Complications of arteriovenous fistulas for hemodialysis," Archives of Surgery, vol. 110, no. 6, pp. 708-712, 1975.

[14] G. E. Pierce, J. H. Thomas, and J. R. Fenton, "Novel repair of venous aneurysms secondary to arteriovenous dialysis fistulae," Vascular and Endovascular Surgery, vol. 41, no. 1, pp. 55-60, 2007.

[15] G. S. Georgiadis, M. K. Lazarides, S. A. Panagoutsos et al., "Surgical revision of complicated false and true vascular access-related aneurysms," Journal of Vascular Surgery, vol. 47, no. 6, pp. 1284-1291, 2008.

[16] H. M. Burkhart and D. F. Cikrit, "Arteriovenous fistulae for hemodialysis," Seminars in Vascular Surgery, vol. 10, no. 3, pp. 162-165, 1997.

[17] M. L. Mantha, R. Baer, G. S. Bailey, R. L. Wu, and J. P. Killen, "Endovascular repair of a hemodialysis fistula aneurysm with covered stents," Kidney International, vol. 76, no. 8, p. 918, 2009.

[18] T. Lee and P. Roy-Chaudhury, "Advances and new frontiers in the pathophysiology of venous neointimal hyperplasia and dialysis access stenosis," Advances in Chronic Kidney Disease, vol. 16, no. 5, pp. 329-338, 2009.

[19] P. Roy-Chaudhury, B. S. Kelly, M. A. Miller et al., "Venous neointimal hyperplasia in polytetrafluoroethylene dialysis grafts," Kidney International, vol. 59, no. 6, pp. 2325-2334, 2001.

[20] R. P. Campos, M. M. Do Nascimento, D. C. Chula, D. E. Do Nascimento, and M. C. Riella, "Stenosis in hemodialysis arteriovenous fistula: evaluation and treatment," Hemodialysis International, vol. 10, no. 2, pp. 152-161, 2006.

[21] P. Goldwasser, M. M. Avram, J. T. Collier, M. A. Michel, S. A. Gusik, and N. Mittman, "Correlates of vascular access occlusion in hemodialysis," American Journal of Kidney Diseases, vol. 24, no. 5, pp. 785-794, 1994.

[22] R. A. Bonomo, D. Rice, C. Whalen, D. Linn, E. Eckstein, and D. M. Shlaes, "Risk factors associated with permanent access-site infections in chronic hemodialysis patients," Infection Control and Hospital Epidemiology, vol. 18, no. 11, pp. 757-761, 1997.

[23] I. Saeed Abdulrahman, S. H. Al-Mueilo, H. A. Bokhary, G. Ladipo, and A. Al-Rubaish, "A prospective study of hemodialysis access-related bacterial infections," Journal of Infection and Chemotherapy, vol. 8, no. 3, pp. 242-246, 2002.

[24] K. B. Stevenson, E. L. Hannah, C. A. Lowder et al., "Epidemiology of hemodialysis vascular access infections from longitudinal infection surveillance data: predicting the impact of NKF-DOQI clinical practice guidelines for vascular access," American Journal of Kidney Diseases, vol. 39, no. 3, pp. 549$555,2002$.

[25] J. I. Tokars, E. R. Miller, and G. Stein, "New national surveillance system for hemodialysis-associated infections: initial results," American Journal of Infection Control, vol. 30, no. 5, pp. 288-295, 2002.

[26] G. Taylor, D. Gravel, L. Johnston et al., "Prospective surveillance for primary bloodstream infections occurring in Canadian hemodialysis units," Infection Control and Hospital Epidemiology, vol. 23, no. 12, pp. 716-720, 2002.

[27] N. R. Powe, B. Jaar, S. L. Furth, J. Hermann, and W. Briggs, "Septicemia in dialysis patients: incidence, risk factors, and prognosis," Kidney International, vol. 55, no. 3, pp. 1081-1090, 1999.

[28] J. H. Tordoir, J. M. Herman, T. S. Kwan, and P. M. Diderich, "Long-term follow-up of the polytetrafluoroethylene (PTFE) prosthesis as an arteriovenous fistula for haemodialysis," European Journal of Vascular Surgery, vol. 2, no. 1, pp. 3-7, 1988.

[29] A. M. Verhallen, M. P. Kooistra, and B. C. van Jaarsveld, "Cannulating in haemodialysis: rope-ladder or buttonhole technique?” Nephrology Dialysis Transplantation, vol. 22, no. 9, pp. 2601-2604, 2007.

[30] J. Tordoir, B. Canaud, P. Haage et al., "EBPG on Vascular Access," Nephrology Dialysis Transplantation, vol. 22, supplement 2, pp. ii88-ii117, 2007.

[31] M. McCann, H. Einarsdóttir, J. P. Van Waeleghem, F. Murphy, and J. Sedgewick, "Vascular access management 1: an overview," Journal of Renal Care, vol. 34, no. 2, pp. 77-84, 2008.

[32] G. Di Minno, J. Martinez, M. L. McKean, J. De La Rosa, J. F. Burke, and S. Murphy, "Platelet dysfunction in uremia. Multifaceted defect partially corrected by dialysis," American Journal of Medicine, vol. 79, no. 5, pp. 552-559, 1985.

[33] G. Eknoyan and C. H. Brown III, "Biochemical abnormalities of platelets in renal failure. Evidence for decreased platelet serotonin, adenosine diphosphate and $\mathrm{Mg}$-dependent adenosine triphosphatase," American Journal of Nephrology, vol. 1, no. 1, pp. 17-23, 1981.

[34] G. Eknoyan, S. J. Wacksman, H. I. Glueck, and J. J. Will, "Platelet function in renal failure," New England Journal of Medicine, vol. 280, no. 13, pp. 677-681, 1969.

[35] G. Remuzzi and F. Pusineri, "Coagulation defects in uremia," Kidney International, vol. 24, pp. S13-S17, 1988.

[36] V. T. Turitto and H. J. Weiss, "Red blood cells: their dual role in thrombus formation," Science, vol. 207, no. 4430, pp. 541-543, 1980 .

[37] D. T. Domoto, J. E. Bauman, and J. H. Joist, "Combined aspirin and sulfinpyrazone in the prevention of recurrent hemodialysis vascular access thrombosis," Thrombosis Research, vol. 62, no. 6, pp. 737-743, 1991.

[38] R. Sreedhara, J. Himmelfarb, J. M. Lazarus, and R. M. Hakim, "Anti-platelet therapy in graft thrombosis: results of a prospective, randomized, double-blind study," Kidney International, vol. 45, no. 5, pp. 1477-1483, 1994.

[39] J. S. Kaufman, T. Z. O’Connor, J. H. Zhang et al., "Randomized controlled trial of clopidogrel plus aspirin to prevent hemodialysis access graft thrombosis," Journal of the American Society of Nephrology, vol. 14, no. 9, pp. 2313-2321, 2003.

[40] M. A. Crowther, C. M. Clase, P. J. Margetts et al., "Lowintensity warfarin is ineffective for the prevention of PTFE graft failure in patients on hemodialysis: a randomized controlled trial," Journal of the American Society of Nephrology, vol. 13, no. 9, pp. 2331-2337, 2002.

[41] M. Israel, "Depression in dialysis patients: a review of psychological factors," Canadian Journal of Psychiatry, vol. 31, no. 5, pp. 445-451, 1986. 
[42] R. S. Stewart, "Psychiatric issues in renal dialysis and transplantation," Hospital and Community Psychiatry, vol. 34, no. 7, pp. 623-628, 1983.

[43] N. Kuroda, K. Saitoi, A. Takada et al., "Suicide by self-stabbing in the city of Tokyo-a review of accumulated data from 1976 to 1995," Japanese Journal of Legal Medicine, vol. 51, no. 4, pp. 301-306, 1997.

[44] T. Karlsson, "Homicidal and suicidal sharp force fatalities in Stockholm, Sweden. Orientation of entrance wounds in stabs gives information in the classification," Forensic Science International, vol. 93, no. 1, pp. 21-32, 1998.

[45] T. Karlsson, K. Ormstad, and J. Rajs, "Patterns in sharp force fatalities - a comprehensive forensic medical study: part 2. Suicidal sharp force injury in the Stockholm area 1972-1984," Journal of Forensic Sciences, vol. 33, no. 2, pp. 448-461, 1988. 


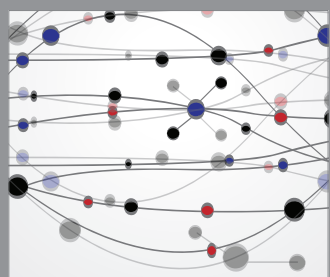

The Scientific World Journal
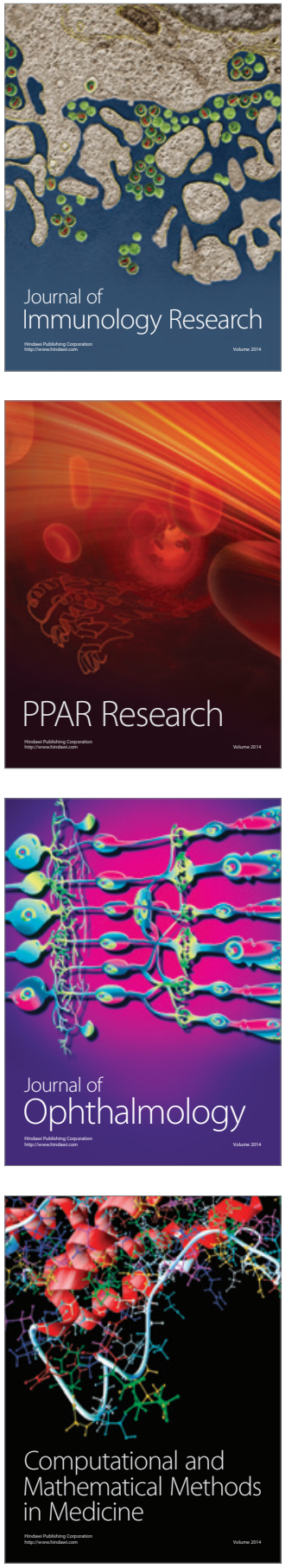

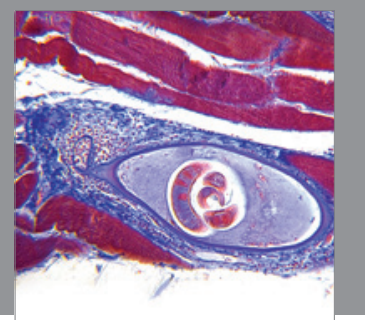

Gastroenterology

Research and Practice
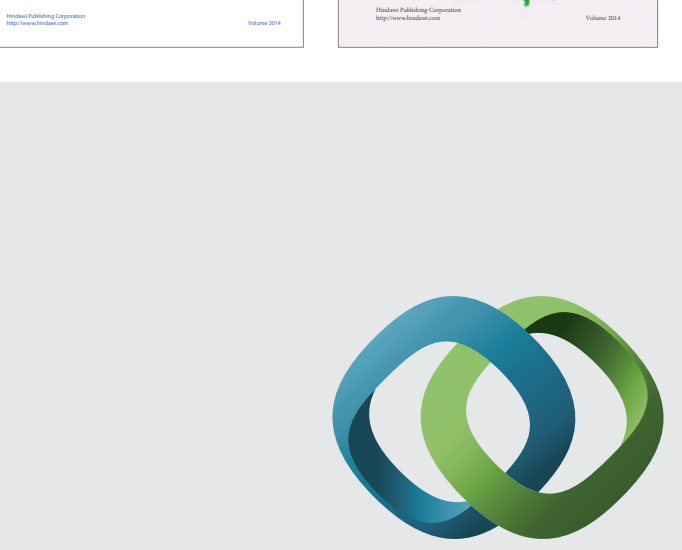

\section{Hindawi}

Submit your manuscripts at

http://www.hindawi.com
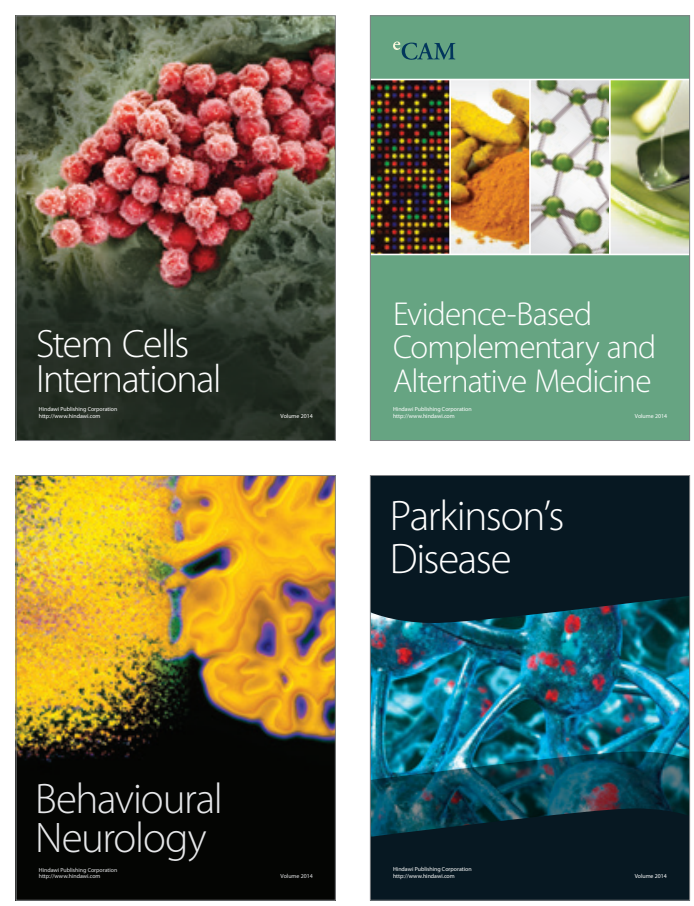

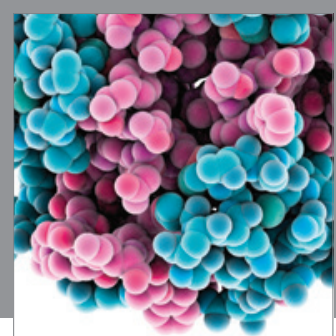

Journal of
Diabetes Research

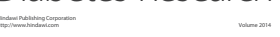

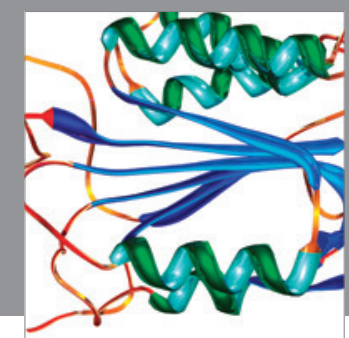

Disease Markers
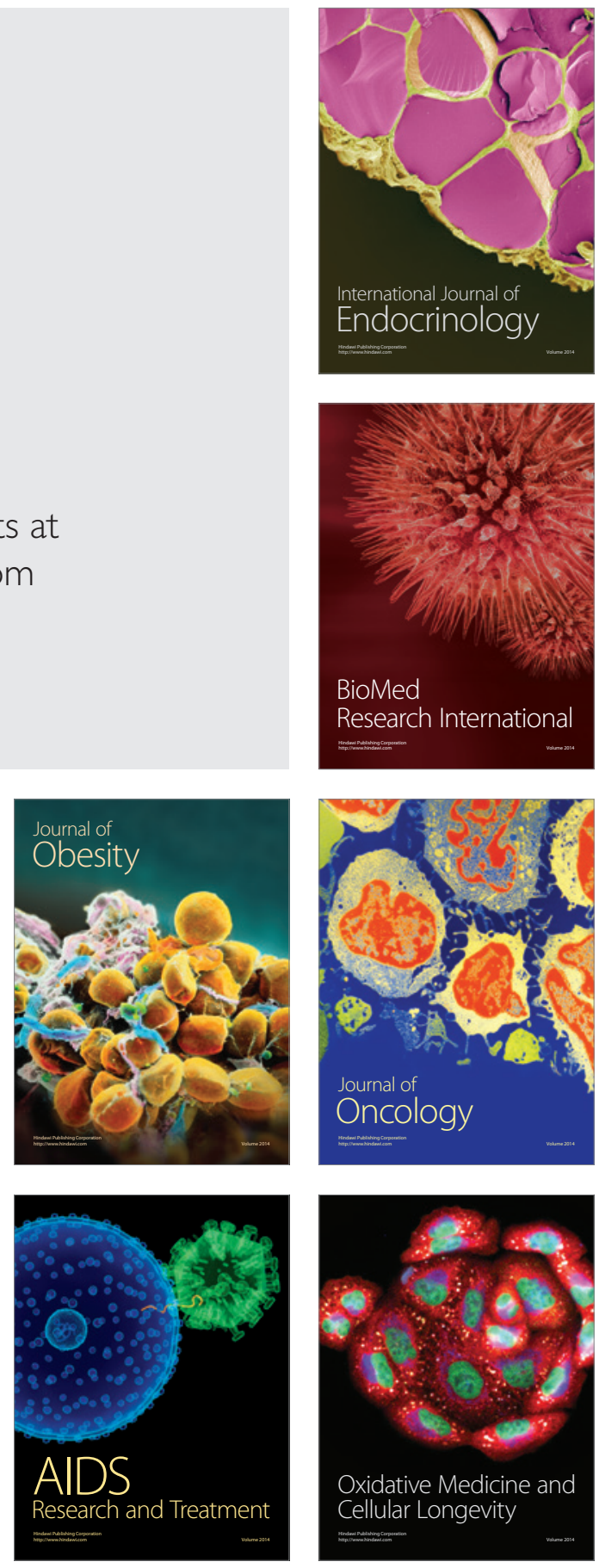